\title{
System-level barriers to personal recovery in mental health: qualitative analysis of co-productive narrative dialogues between users and professionals
}

\author{
Miharu Nakanishi*, George Kurokawa*, Junko Niimura, Atsushi Nishida, Geoff Shepherd and \\ Syudo Yamasaki
}

\section{Background}

No co-productive narrative synthesis of system-level facilitators and barriers to personal recovery in mental illness has been undertaken.

\section{Aims \\ To clarify system-level facilitators and barriers to personal recovery of people with mental illness.}

\section{Method}

Qualitative study guided by thematic analysis. Data were collected through one focus group, which involved seven service users and three professionals. This group had 11 meetings, each lasting $2 \mathrm{~h}$ at a local research institute, between July 2016 to January 2018

\section{Results}

The analysis yielded three themes: barriers inhibiting positive interaction within personal relationship networks, roots of barriers from mental health systems and the social cultural context and possible solutions to address the roots. Barriers were acknowledged as those related to sense of safety, locus of control within oneself and reunion with self. The roots of barriers were recognised within mental health services, including system without trauma sensitivity, lack of advocacy support and limited access to psychosocial approaches. Roots from social cultural context were also found. There were no narratives relating to facilitators. A possible solution was to address the roots from systems. Social cultural change was called for that makes personalised goals most valued, with an inclusive design that overcomes stigma, to achieve an open and accepting community.

\section{Conclusions}

The analysis yielded system-level barriers specific to each recovery process. Roots of barriers that need transformation to facilitate personal recovery were identified within mental health services. Social interventions should be further explored to translate the suggested social cultural changes into action.

\section{Keywords}

Consumer advocacy; co-production; mental health recovery; narrative medicine; qualitative research.

\section{Copyright and usage}

(C) The Author(s), 2021. Published by Cambridge University Press on behalf of the Royal College of Psychiatrists. This is an Open Access article, distributed under the terms of the Creative Commons Attribution-NonCommercial-NoDerivatives licence (http://creativecommons.org/licenses/by-nc-nd/4.0/), which permits non-commercial re-use, distribution, and reproduction in any medium, provided the original work is unaltered and is properly cited. The written permission of Cambridge University Press must be obtained for commercial re-use or in order to create a derivative work.
Mental healthcare is encouraged to support personal recovery of people living with mental illness. Personal recovery is a process whereby people pursue life goals in the spirit of hope despite mental health symptoms. ${ }^{1-4}$ Personal recovery is not only based on symptom remission or disappearance, but is a lifelong process toward being a functional, resilient and happy individual. Supporting recovery in mental health systems is a global imperative. ${ }^{5}$ Some recovery-oriented services have shown an improvement in main outcomes of service users; ${ }^{3,6-8}$ however, there may be system-level barriers to the implementation of supporting recovery in mental health services. Understanding system-level facilitators and barriers is a crucial step to establishing a recovery-oriented mental health system. A recognition gap about facilitators and barriers remains between users and care professionals. From a service user perspective, their psychosocial strengths, such as learning and willpower, ${ }^{9}$ and perception of recovery identification, ${ }^{10}$ were identified as facilitators, whereas negative effects of relationships with mental health professionals were acknowledged as barriers., ${ }^{9,11}$ Contrarily, mental healthcare professionals recognised both facilitators and barriers in their organisational culture toward supporting

* Joint first authors. recovery. ${ }^{12,13}$ Co-production, where work is shared between users and professionals, is at the heart of commissioning and service design. ${ }^{14}$ Therefore, a co-productive investigation can contribute to identifying and sharing the basis of a strong therapeutic alliance aimed at achieving personally meaningful goals for recovery. Coproducing interactive interviews may yield mutual and reciprocal understanding, and unexpected insights into mental health service improvements. Such an understanding has further implications for global strategies of supporting recovery in mental health systems. However, to our knowledge, no co-productive narrative synthesis of system-level facilitators and barriers to personal recovery has been undertaken.

Japanese mental healthcare also has a recovery-oriented system that needs further development. The Japanese mental healthcare system has predominantly remained dependent on hospital-based services, and community mental health services are not well-developed compared with other countries within the Organisation for Economic Co-production and Development. ${ }^{15}$ Users often suffer from a prolonged length of stay at psychiatric hospitals because of a lack of community mental health services. ${ }^{16}$ Japanese mental health practices also include involuntary admissions and the use of coercion (e.g. seclusion and physical restraint). ${ }^{17,18}$ Currently, most of the existing evidence for personal recovery is based on 
European and Northern American studies. ${ }^{19,20}$ Findings from Japan will support the need for establishment of recovery-oriented mental healthcare systems. ${ }^{21}$ The present study aimed to clarify systemlevel facilitators and barriers to personal recovery of people with mental illness.

\section{Method}

\section{Study design and setting}

Data were collected through a series of discussions with one focus group, with each session lasting $2 \mathrm{~h}$. The sessions took place by arrangement at a local research institute. The focus group, involving 7 users and 3 professionals, had 11 meetings during the 19-month period from 30 July 2016 to 13 January 2018. Having a series of discussions with a single focus group was decided, to take time to build positive and trusting relationships among all members, which is a fundamental need for co-production. ${ }^{22}$ The series was concluded at 11 th meeting because the participants agreed that they had covered all components for each theme.

The authors assert that all procedures contributing to this work comply with the ethical standards of the relevant national and institutional committees on human experimentation and with the Helsinki Declaration of 1975, as revised in 2008. Ethical approval was granted by the ethics review board at the Tokyo Metropolitan Institute of Medical Science (approval numbers 16-37, 16-38 and 19-28). Information sheets regarding the nature, purpose and requirements of the study were given to potential participants, who were asked to contact the lead researcher if they wished to participate. Written consent was obtained from all participants, who were reassured that they would remain anonymous in the reporting of the study, and that they could leave the study at any point.

\section{Participants}

We used the method of snowball and convenience sampling, which is a commonly used sampling approach in sociology. ${ }^{23}$ Given that co-producing dialogues, where users and professionals use the same language and terminology as each other, are rare and innovative in mental healthcare, snowball sampling was appropriate, and other methods would have been difficult to use to recruit our participants. The key characteristics of participants are presented in Table 1.

\section{Service users}

Our inclusion criteria for the study were service users in contact with the local mental health voluntary sector. Peer support workers asked service users in routine appointments if they would be interested in participating. In total, seven service users were recruited; all seven were men.

\section{Mental health professionals}

Our inclusion criteria for mental healthcare professionals were that they were working in community mental health teams or who had been doing similar work in the same catchment area. In total, three professionals were recruited (a psychologist, nurse and volunteer worker); there were two men and one woman. We did not recruit psychiatrists based on a previous experience of co-production in the 'Healthy Active Lives in Japan' workshop meeting, where some users tended to avoid disclosing negative experiences of mental health services in front of psychiatrists. ${ }^{24}$ The avoidance to share own experiences with psychiatrists was also found in another Japanese report. ${ }^{25}$

\section{Data collection}

The focus group discussion series was conducted by S.Y. (psychologist) and G.K. (user), following a flexible topic guide that explored participants' background experience in mental health services, understanding of mental health and personal recovery. The topic guide was developed by S.Y., G.K. and J.N. (nurse), to initiate the discussion series with sharing general knowledge and literature on the recovery process ${ }^{2}$ between users and professionals. A nonjudgemental, open question style was adopted, and participants were encouraged to introduce issues of importance not covered by the topic guide. Discussion meetings were audio-recorded and transcribed verbatim. Identifying information was removed at transcription, after which recordings were destroyed. Meeting duration averaged $145.3 \mathrm{~min}$ (range 112-185 min). Theme generation and analysis occurred after completion of data collection.

\section{Analysis}

Data were analysed by an inductive thematic analysis that offers a systematic method of identifying patterns across the data corpus. ${ }^{26}$ Initial familiarisation with the data was achieved by reading each transcript several times, followed by manual line-byline coding, and final clustering and synthesis of codes to form themes. Both analysts, one who did not participate in the discussion series (M.N.) and another who did participate in the discussion series (S.Y.), read all transcripts, with input from the wider research team, who met regularly to review and discuss the evolving analysis. The team comprised academic researchers and clinical professionals, three of whom had personal experience of psychiatric inpatient and out-patient treatment. Any disagreements on the themes identified between analysts were resolved through discussion with independent experts and resolved through consensus. Participating users were also invited to comment on the analysis. This ensured that the themes were represented in the source material.

\begin{tabular}{|c|c|c|c|c|}
\hline Number & Type & Age, years & Gender & Experience with mental health services \\
\hline 1 & User & $40 \mathrm{~s}$ & Man & Person with major depressive disorder and a 20-year experience as a service user \\
\hline 2 & User & $40 \mathrm{~s}$ & Man & Person with obsessive-compulsive disorder and a 25-year experience as a service user \\
\hline 3 & User & $30 \mathrm{~s}$ & Man & Person with depression and a 5-year experience as a service user \\
\hline 4 & User & $40 \mathrm{~s}$ & Man & Person with schizophrenia and a 25-year experience as a service user \\
\hline 5 & User & $40 \mathrm{~s}$ & Man & Person with schizophrenia and a 15-year experience as a service user \\
\hline 6 & User & $40 \mathrm{~s}$ & Man & Person with schizophrenia and a 20-year experience as a service user \\
\hline 7 & User & $30 \mathrm{~s}$ & Man & Person with schizophrenia and a 15-year experience as a service user \\
\hline 8 & Professional & $30 \mathrm{~s}$ & Man & Psychologist at a day care centre affiliated with the university hospital \\
\hline 9 & Professional & $50 \mathrm{~s}$ & Woman & Nurse at a public health centre \\
\hline 10 & Professional & $30 \mathrm{~s}$ & Man & Volunteer worker at a private social welfare corporation \\
\hline
\end{tabular}




\section{Results}

Three themes were identified, as given in Fig. 1. Barriers to recovery (theme 1) was acknowledged as those inhibiting positive interaction within one's personal relationship network during the recovery processes that oriented rebuilding a sense of continuity in life, before and after developing mental illness. Barriers had roots (theme 2) in the mental health systems and social cultural context. A possible solution to address the roots (theme 3) was identified as organisational and social cultural changes. First, we will outline each theme, as well as provide descriptions of subthemes (Supplementary Appendix 1 available at https://doi.org/10.1192/bjo.2020.156). We will then present several quotes as instances.

\section{Theme 1: barriers to recovery}

From the onset of mental illness, the user feels outside of the life course that they used to traverse. The user recognises themself as being far away from others who go along 'normal' roads. When the user wishes to return to themselves without mental illness, the person often suffers from a sense of discontinuity in their own life. If the user finds themselves by interaction within their personal relationship network, the person can recognise themself as being as human as others without mental illness, and recover a sense of continuity in their own life. Thus, system-level barriers were acknowledged as those inhibiting the positive interaction.

'Personal recovery would not start while the user keeps questioning "why did I have to be sick". It starts when the user accepts him/herself as a person who has mental illness, and has the natural energy to direct towards new goals or something to do' (User 1)

\section{Sense of safety}

Recovery processes were identified as starting from 'sense of safety'. Sense of safety further contained 'psychological safety' and 'human rights'. Barriers to safety included 'lack of compassion' and 'life security is threatened'.

Lack of compassion. The recovery process starts when the user has a sense of being accepted in the community regardless of having mental illness. To ensure the user's psychological safety, mental healthcare should avoid re-traumatisation. However, mental health professionals sometimes exhibit a lack of compassion toward the lived experiences of users.

'We have launched the HeAL (Healthy Active Lives) Japan Initiative because psychiatrists generally pay little attention to the overall health of people with mental illness. Our participants at the symposium reported that psychiatrists did not listen with empathy to the users' voice and their concerns about psychotropic medications' (Psychologist 1).

'Without a viewpoint based on personal recovery in mind, mental health professionals and/or people surrounding the person would start taking control of emotion and mood over the user rather than listening empathetically to the user's problems' (User 1).

Life security is threatened. Life security provides the basis for an individual's journey of personal recovery. The users would lose equal access to education or work because of having a mental illness, which can lead to poverty in later life. Family members are often the last resource of care and financial support.

'Users who live on social security have little financial capacity and feel uncertain about their own lives after their parents eventually pass away in the future. They have nothing but despair, feel isolated and left behind' (User 2).

'The lack of money limits our choices in lives, resulting in limited ability and capacity to tackle some challenges on our own' (User 3).

\section{Locus of control within oneself}

'Locus of control within oneself followed 'sense of safety' in the recovery processes. Locus of control consisted of 'choice is available', 'shared decision-making' and 'self-efficacy'. Barriers to control included 'choice is unavailable', 'authority slope' and 'psychosis and antipsychotics'.

Choice is unavailable. The recovery process involves the user having a sense of control. Choice should be available at any time to promote control. Current mental health systems require the user to follow pre-defined treatment protocols and procedures.

'It is all right if the user is struggling with approaches to how to individualise care and support which is available under the current mental health systems. However, many users cannot even seek out choices of mental healthcare' (User 2).

'I think peer staff can help users to choose appropriate professional service via clarifying unmet needs and possible pros and cons of the service. If professional staff suddenly told the user "you can choose freely" without any preparation, users may hardly be able to choose the appropriate service for themselves' (Psychologist 1).

Authority slope. Shared decision-making helps the user recover a sense of self. Meanwhile, users find it challenging to disclose their own experiences with mental illness to mental health professionals. Any chance for consultation and discussion of treatment choices, including use of medication and type of medication, is usually unavailable.

'In a conversation with psychiatrists, users are led to respond to simplified and typical questions, such as "do you sleep enough these days?" although they try to disclose their own experiences with mental illness to professionals. They also feel themselves "labelled" or "exploited" as a source of information' (Volunteer Worker 1).

'In the past, psychiatric professionals in Japan have overwhelmingly suppressed (autonomy of) service users. What they have done as "treatment" are means to control user's behaviour' (User 1).

Disempowerment owing to symptoms and medication. Users could not trust their own feelings, wishes or abilities to make decisions since developing mental illness. Use of antipsychotics and side-effects might add to distrust in oneself. Under the current Japanese mental health systems, availability of psychosocial approaches and environmental modifications are limited to support self-efficacy of people with mental illness.

'I understand psychotropic medications help me to reduce symptoms and elevate self-efficacy; however, at the same time I feel that my recovery process is sometimes hindered by side-effects of psychotropic medications, such as sleepiness, fatigue and reduced power of thinking' (User 5).

'Psychiatrists who are good at pharmacotherapy acknowledge what they can and cannot do with medication. They delegate to other professional staff what the medication cannot address. I can trust them very much' (Psychologist 1 ).

\section{Reunion with self}

'Reunion with self' followed 'locus of control within oneself' in the recovery processes. Reunion with self included 'be a part of society' 


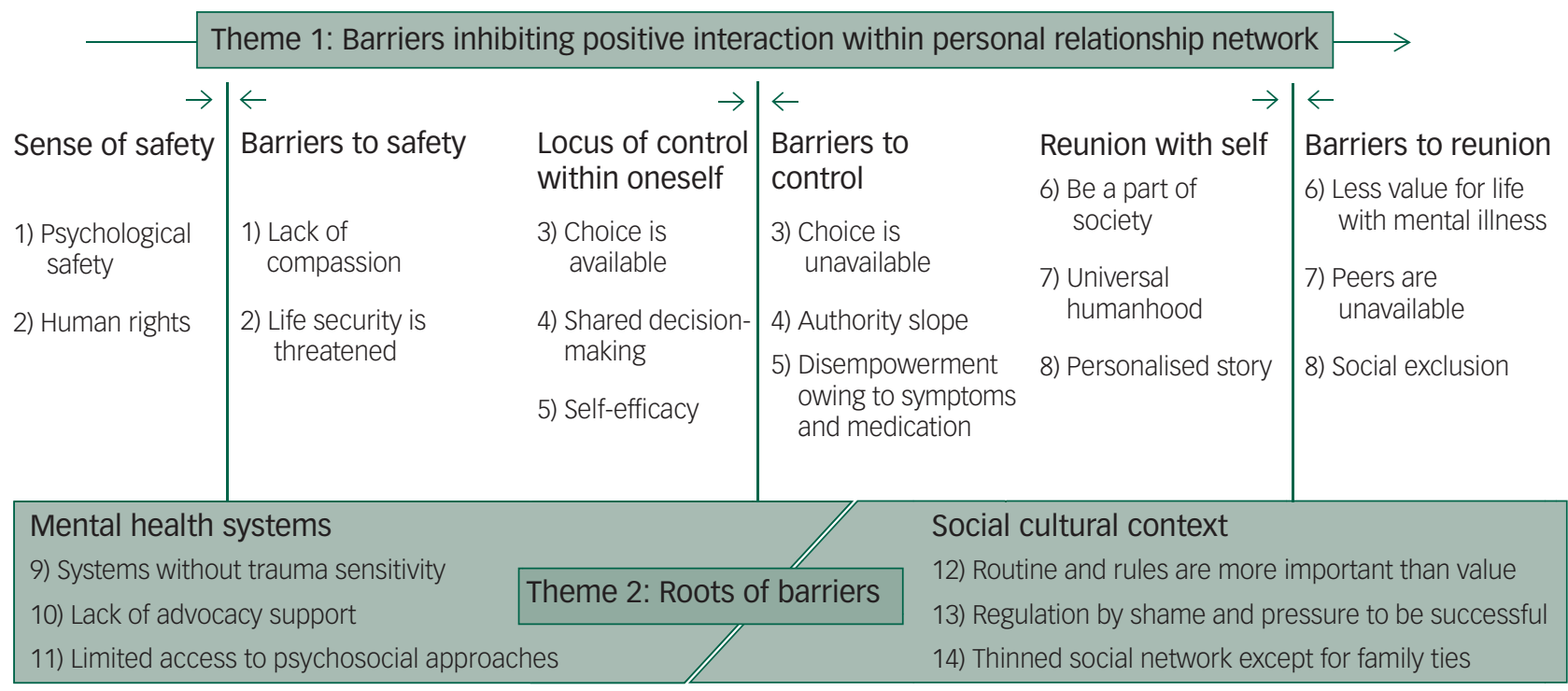

\begin{tabular}{|l|l|}
\hline Organisational change to resolve & Social cultural change to resolve \\
9) Trauma-informed care systems & 12) Personalised goal is most valued \\
$\begin{array}{ll}\text { 10) Advocacy support available in crisis } \\
\text { 11) Community- and network-based structure }\end{array}$ & 13) Inclusive design that overcomes stigma \\
\hline
\end{tabular}

Fig. 1 System-level barriers to personal recovery, roots of barriers and solutions.

and 'universal humanhood'. Barriers to reunion contained 'less value for life with mental illness' and 'peer is unavailable'.

Less value for life with mental illness. A person finds their own identity from interaction within their personal relationship network. Users can have their own unique role, mission and values within the network as a part of society. However, when having mental illness, users feel they are outside of society.

'When the Tohoku earthquake and tsunami affected Japan [11 March 2011], mutual support in personal relationship networks became increasingly important. Meanwhile, the general public addressed people with mental illnesses and disabilities very differently. The perpetrator of the Sagamihara stabbings [26 July 2016] made the statement that people with challenges should die because they have no role in society. There were a certain number of people active online who indicated their agreement and regarded him as a hero' (User 5).

Peers are unavailable. The user identifies someone who has experiences with mental illness as a role model. The role model provides a source of hopefulness about being a part of society alongside people without mental illness. Current mental health systems often fail to offer chances or a place to acknowledge users' peers. Peers are unavailable because of social exclusion of the users from society, little capacity to have peer staff in mental health organisations or unsolicited roles of peer staff.

'Users have little opportunity to acknowledge peers who have mental illness and who are working as a part of community, serving as a role model of personal recovery. Therefore, they lack the reflections for what they can achieve and what challenges they may face. The lack hinders personal recovery' (Psychologist 1).
'If an organisational system is not established in which everyone works together to ease part of the burden on peer staff, their work will not be successful due to a disproportional workload on peer staff (User 2).

Social exclusion. The user takes a unique journey for recovery, as every person is unique. The unique relation network can both hurt and heal the user. The user has a right to take own risk as well as other people do. However, having mental illness would cause exclusion of the user from communities and relationships that belonged to the person.

'I feel like my life with mental illness is too hard, being affected by the public stigma against people with mental illness, and our society as a difficult one to live in. So I want to change the society, to make the public have better understanding of mental illness, and free from preconceived notions of mental illness' (User 5).

'People with mental illness often feel like being treated as untrustworthy by the society. They tend to self-stigmatise as a result of the stigma in the society' (Psychologist 1).

\section{Theme 2: roots of barriers}

Mental health systems

Systems without trauma sensitivity. Most users have traumatising experiences before the onset of mental illness. However, current mental health systems without trauma sensitivity could cause retraumatisation that threatens psychological safety. An authority slope usually exists between mental health professionals and users. Some organisations have peer staff; however, the directors do not intend to lead organisational changes.

'Many patients in psychiatric hospitals, who have traumatising experiences, receive coercive treatments that lead to re- 
traumatisation' (Nurse 1).

'Even community services, such as outreach nursing care providers, tend to select cancer patients and avoid those with mental illness in order to make their business stable' (Nurse 1). 'Psychiatric hospitals explain that they cannot hire peer staff because the safety of peer staff cannot be ensured. However, if hospital staff are prepared to do so, I believe they would be able to work with peer staff in a safe manner' (User 1).

Lack of advocacy support. Compulsory admission is frequently adopted in mental healthcare. Advocacy support is unavailable at crisis and psychiatric hospital admission. Medication is often started without the user's agreement. The lack of advocacy support disables choices, shared decision-making and the user's self-efficacy. There might be some overlap with 'system without trauma sensitivity', although 'lack of advocacy support' was focused on support at crisis rather than whole process of mental health services.

'Some users who have had several psychiatric admissions experienced re-traumatisation during hospitalisation. While it depends on the quality of care, hospitalisation may lead the user to re-experience the past if coercive measures, such as physical restraint, were used during previous in-patient care' (Psychologist 1).

'Until now, professionals have targeted expressed behaviours of users, without looking at what they have experienced. If the person with mental illness expressed shouting, they treat him only by suppressing the behaviour with drugs' (User 1).

Limited access to psychosocial approaches. Mental health professionals do not make appropriate referrals to social services even when the life security of the user is threatened. Mental health professionals stick to medical practices that are refundable under the benefit schedule for public healthcare insurance programme. They tend to pay little attention to the personal relationship network of which the user is a part. They treat family members of the user as a source of caregiving rather than persons who have individual needs and stories.

'There is a lack of function that bridges the barriers between hospital and community mental health services. It is undetermined who is expected to perform the bridging role in relationship networks, community service providers or hospital case managers' (User 1).

'The old-fashioned doctors seem to seek any approaches within the hospital. Even after the patients with mental illness are discharged from the hospital, they appear to address everything within hospital-affiliated out-patient service only' (Nurse 1).

\section{Social cultural context}

Routines and rules are more important than value. People share the cultural norm that routines and rules should be followed at any time. Little importance is attached to personal wishes and values. Health and social care services are entirely defined by the universal benefit schedule. This combination of culture and structure makes mental health services inflexible, compelling the user to follow a solicited treatment protocol.

'I felt uncomfortable within existing social care services. Their work style leans to "management" of us, or to treating us as if we were children' (User 3).

'Even though we are professionals, we have little control for own working hours and no choice but to do as our boss orders. We need a working system that enables us to negotiate with the organisation for more flexibility' (Nurse 1).

Regulation by shame and pressure to be successful. Human behaviours are regulated by a sense of shame and pressure to be successful. A man is valued only when he has earnings to support his wife and children. A woman is valued only when she has her husband and children to take care of. The user would feel shame and guilt about failing to meet these expectations. Society uses shame and pressure to force people to be 'productive'. Society is not designed to include various people with challenges or diverse backgrounds such as minorities.

'We may have mixed feelings of fault and hurt. Although the user has not done anything wrong, there is a sense of guilty conscience, own fault, shame, and self-discrimination. We share the fear of being rejected by society' (User 1).

'I feel the strong influence of culture ... in family relationship or community on users. People with mental illness will not be able to say what they want to say under the belief that having a mental illness is a shame' (Nurse 1).

Thinned social network except for family ties. Most people (in Japan) have no specific belonging groups outside work or family, such as charity organisations or those based on religious beliefs. Society thus lacks support groups based in communities. There would be few safe rooms in society where anyone is accepted and valued as a person. Family ties are deemed as a last resort, although many users have had complex traumas in family relationships.

'This is common in male, older adults. They spend their life exclusively on working at a job, and reach the age of retirement. After their wife passes away, they face huge hardship in their lives. The reason is that they have never learned about how to manage their social life outside the workplace' (Psychologist 1 ). 'We want a place where people from different walks of life can come together, exchange and share their thoughts on an equal basis' (User 2).

\section{Theme 3: solution to address roots of barriers}

\section{Organisational change}

Trauma-informed care systems. Participants acknowledged that mental health services should be transformed into trauma-informed care systems.

'Mental health professionals should have attributes and abilities to recognise the characteristics of (trauma) experiences that hold the user inside and hardly let the person leave' (User 1).

'A flexible environment is critical to build mutual trust between people with mental illness and professionals. But in a hospital-centred mental healthcare system, I think we are at a great disadvantage in creating such an environment' (Psychologist 1).

Advocacy support available in crisis. Peer support during a crisis was suggested as a possible solution to overcome challenges owing to lack of advocacy support.

'The main strength of peer staff might be their ability to provide connectedness and a therapeutic alliance to the user at first contact with mental healthcare and professionals' (Psychologist 1).

'Peer staff try to build mutual trust and value the relationship with the users. The valued relationship enables us to understand comfortable approaches for the users, and to respond appropriately to their questions and concerns' (User 1).

Community- and network-based structure. Current mental health systems in Japan were recognised as established in hospital- and medication-centred structures. The structure might limit access to psychosocial approaches. Participants suggested a transformation of mental health systems toward community- and network-based structures. 
'Mental health professionals should actively seek collaboration with community mental health services to extend social care services, which cannot be fulfilled within medical practices. To enable this collaboration, mental health systems require system-level transformation' (User 1).

'Needs and circumstances vary among people with mental illness, and the needs even vary in the same person at different stages. We would like to make more options available for each person. Recovery College would be a good example that is flexible and tailored to the parties involved' (Psychologist 1).

\section{Social cultural change}

Personalised goals are most valued. Participants suggested a counter-culture that would reform the social cultural norms on which current mental health services stand. The culture most values personalised goals. These were seen in some peer-to-peer activities.

\section{'Supported workplaces under current social care systems provide users with little choice. We work in a responsive manner, resulting in the feeling that we are compelled to do that work. Peer-to-peer activities are appealing for me, because we can make our own choices in a voluntary, proactive manner, based on our own wishes. This means that I am my own boss' (User 5). \\ 'Mutual communication should be encouraged between people with and without mental illness to exchange unique experi- ences and values with each other' (Psychologist 1).}

Inclusive design that overcomes stigma. The users noted the importance of peer support among people with mental illness, as it posed an example of inclusive design of the whole society that overcomes stigma toward people with diverse challenges.

'Management of organisations only by members with mental illness does not work well as people without mental illness would feel uncomfortable to come in. Joint commitment is vital' (Psychologist 1).

Open and acceptable community. Participants also requested a social cultural change toward open and acceptable community, to increase the small number of safety rooms in society where anyone is accepted and valued as a person.

'We-people with mental illness - should participate in a movement toward establishment of a community that accepts people with challenges' (User 1).

'I agree that the binary opposition between peer staff and professional should be avoided' (Psychologist 1).

\section{Discussion}

\section{Summary of findings}

The present study undertook a co-productive narrative synthesis of system-level facilitators and barriers to personal recovery among people with mental illness. System-level barriers to recovery were acknowledged as those inhibiting positive interaction within personal relationship networks in the recovery processes. Roots of barriers were recognised within mental health systems and the social cultural context. No system-level facilitators for recovery were identified. Instead, organisational and social cultural changes were identified as possible solutions to address the roots of barriers.

\section{Comparison with wider literature}

The thematic analysis in our data yielded barriers to recovery that were specific to each recovery process among people with mental illness. The present study newly identified roots of barriers, which may have been described as barriers to recovery in previous studies. For example, 'systems without trauma sensitivity' may overlap with 'competing priorities' in mental health systems ${ }^{12}$ and organisational culture. ${ }^{13}$ 'Routine and rule is more important than value' would overlap with stigma as a barrier to recovery for people with mental illness. ${ }^{27}$ It should be noted that our study focused on system-level barriers and the aim of the study was shared with participating users and professionals. This approach could have helped participants to not attribute barriers to patient characteristics and personal traits. The discussion series started with sharing of literature on the recovery process ${ }^{2}$ among the participants. This was done to simply set the stage for detailed discussion between users and professionals. It was the personal stories and views of the participants that promoted our understanding of an explicit whole structure of system-level barriers based on the shared knowledge about personal recovery.

No system-level facilitators were identified in this study, although previous research suggested some facilitators, such as learning, social relations and willpower of service users, ${ }^{9}$ and competences of mental healthcare providers. ${ }^{28}$ The role of peer support has been emphasised in working with these facilitators. ${ }^{4,29}$ However, our participants described the interaction with peers as a recovery process in itself rather than a facilitator. One possible explanation is that there was a lack of clarity on the concept of a 'facilitator'. One other possibility is that there is an actual lack of facilitators as a result of low availability of social relations in the Japanese mental health systems. ${ }^{15}$ Our participants clearly mentioned the lack of social relations when relating situations in which a person with mental illness would have relations only with family members and mental health professionals. Introducing peer support into an organisation requires significant organisational changes in its culture. ${ }^{30}$ Hence, mental healthcare reforms should be further equipped to develop a community- and network-based structure in Japanese mental health organisations, as suggested by the participants. Co-production should be at the heart of such reforms, including design of peer support. ${ }^{14}$ This may also apply to other countries with high need for improvement in mental health outcomes.

The recovery process described in this study was different from the pre-defined recovery process that started at 'connectedness'. Our recovery process started with 'sense of safety', and was followed by 'locus of control within oneself and 'reunion with self. There seemed to be similarities between some categories of 'reunion with self and 'connectedness'; however, the order of the recovery process did not match previous findings. Because our study aimed to elucidate facilitators and barriers but not the recovery process itself, our results may have collated some points of interaction between users and mental health systems, rather than a whole process toward personal recovery.

\section{Strengths and limitations}

The strength of our study lies in the co-productive implications for mental health reforms as well as system-level barriers to personal recovery. Suggested solutions are in alignment with international recommendations ${ }^{31}$ that involve an organisational cultural change. ${ }^{32}$ However, our results would be subject to a gender bias, because service users in the study did not include women with mental illness. The convenience sampling method might lead to recruit service users and professionals who had more favourable attitudes to support recovery. Results should be generalised with caution, especially on the roots of barriers in the social cultural context. A large power distance culture in Japan compared with European countries ${ }^{33}$ may have caused an overreliance on these aspects of the social cultural context. Narratives from psychiatrists 
or other medical doctors were also lacking in the study. Some service users may have been reluctant to self-disclose in front of professionals, as suggested by Japanese reports, ${ }^{24,25}$ despite the fact that our participating professionals were independent from the care team or organisation that provided care for participating users.

\section{Implications}

Our participants suggested key components for mental health systems to address the roots of system-level barriers and social cultural changes. However, implementation approaches of these solutions and social actions remain to be further examined. Future qualitative and quantitative research with a more rigorous design needs to confirm the findings of this study and develop practical approaches to implement these interventions.

Miharu Nakanishi $(\mathbb{D}$, PhD, Research Center for Social Science \& Medicine, Tokyo Metropolitan Institute of Medical Science, Japan; George Kurokawa, Peer Staff Department, Sudachikai Social Welfare Corporation, Japan; Junko Niimura, PhD, Research Center for Social Science \& Medicine, Tokyo Metropolitan Institute of Medica Science, Japan; Atsushi Nishida, PhD, Research Center for Social Science \& Medicine Tokyo Metropolitan Institute of Medical Science, Japan; Geoff Shepherd, PhD, Program Director, Implementing Recovery through Organisational Change (ImROC), UK; Syudo Yamasaki, PhD, Research Center for Social Science \& Medicine, Tokyo Metropolitan Institute of Medical Science, Japan.

Correspondence: Miharu Nakanishi. Email: mnakanishi-tky@umin.ac.jp First received 23 Jul 2020, final revision 30 Nov 2020, accepted 1 Dec 2020

\section{Supplementary material}

Supplementary material is available online at https://doi.org/10.1192/bjo.2020.156.

\section{Data availability}

The data that support the findings of this study are available on request from the corresponding author. M.N. The data are not publicly available because they contain information that could author, M.N. The data are not publicly available becis
compromise the privacy of research participants.

\section{Acknowledgements}

The authors would like to thank the users and professionals who participated in the survey.

\section{Author contributions}

All authors meet all four ICMJE criteria for authorship. M.N. contributed to data analyses and drafted the manuscript. G.K. contributed to the design of the study, recruited participants, supervised data analyses and revised the manuscript. J.N. contributed to participant recruitsupervised data analyses and revised the manuscript. J.N. contributed to participant recruit-
ment and revised the manuscript. A.N. contributed to the design of the study and revised the manuscript. G.S. supervised data analyses and revised the manuscript. S.Y. conceived and designed the study, performed data analyses and revised the manuscript. All authors have approved the final manuscript.

\section{Funding}

The study was funded by a grant-in-aid for challenging exploratory research from the Ministry of Education, Culture, Sports, Science, and Technology of Japan (grant number JP16K13499): of Education, Culture, Sports, Science, and Technology of Japan (grant number JP16K13499);
and by the Ministry of Health, Labour Sciences research grant (grant number 19GC1010) from the Ministry of Health, Labour and Welfare. The funders had no role in the study design, data collection, and analysis, interpretation, decision to publish or preparation of the manuscript.

\section{Declaration of interest}

None.

ICMJE forms are in the supplementary material, available online at https://doi.org/10.1192/ bjo.2020.156

\section{References}

1 Repper J, Perkins R. Social Inclusion and Recovery: A Model For Mental Health Practice. Bailliere Tindall, 2003.

2 Leamy M, Bird V, Le Boutillier C, Williams J, Slade M. Conceptual framework for personal recovery in mental health: systematic review and narrative synthesis. Br J Psychiatry 2011; 199: 445-52.
3 Slade M, Amering M, Farkas M, Hamilton B, O'Hagan M, Panther G, et al. Uses and abuses of recovery: implementing recovery-oriented practices in mental health systems. World Psychiatry 2014; 13: 12-20.

4 Corrigan PW, Larson JE, Smelson D, Andra M. Recovery, peer support and confrontation in services for people with mental illness and/or substance use disorder. Br J Psychiatry 2019; 214: 130-2.

5 World Health Organization (WHO). Mental Health Action Plan 2013-2020. WHO, 2013 (https://apps.who.int/iris/bitstream/handle/10665/89966/9789241506021_ eng.pdf).

6 Slade M, Bird V, Le Boutillier C, Farkas M, Grey B, Larsen J, et al. Development of the REFOCUS intervention to increase mental health team support for personal recovery. Br J Psychiatry 2015; 207: 544-50.

7 Slade M, Bird V, Clarke E, Le Boutillier C, McCrone P, Macpherson R, et al. Supporting recovery in patients with psychosis through care by community-based adult mental teams (REFOCUS): a multisite, cluster, randomised, controlled trial. Lancet Psychiatry 2015; 2: 503-14.

8 Winsper C, Crawford-Docherty C, Weich S, Fenton SJ, Singh SP. How do recovery-oriented interventions contribute to personal mental health recovery? A systematic review and logic model. Clin Psychol Rev 2020; 76: 101815.

9 Petersen KS, Friis VS, Haxholm BL, Nielsen CV, Wind G. Recovery from mental illness: a service user perspective on facilitators and barriers. Community Ment Health J 2015; 51: 1-13.

10 Cruwys T, Stewart B, Buckley L, Gumley J, Scholz B. The recovery model in chronic mental health: a community-based investigation of social identity processes. Psychiatry Res 2020; 291: 113241.

11 Wood L, Alsawy LWS. Recovery in psychosis from a service user perspective: a systematic review and thematic synthesis of current qualitative evidence. Community Ment Health J 2018; 54: 793-804.

12 Whitley R, Gingerich S, Lutz WJ, Mueser KT. Implementing the illness management and recovery program in community mental health settings: facilitators and barriers. Psychiatr Ser 2009; 60: 202-9.

13 Le Boutillier C, Slade M, Lawrence V, Bird VJ, Chandler R, Farkas M, et al. Competing priorities: staff perspectives on supporting recovery. Adm Policy Ment Health 2015; 42: 429-38.

14 Skills for Care. Co-production in Mental Health: Not Just Another Guide. Skills for Care, 2018 (https://www.skillsforcare.org.uk/Documents/Topics/Mentalhealth/Co-production-in-mental-health.pdf).

15 Organisation for Economic Co-operation and Development (OECD). OECD Reviews of Health Care Quality: Japan. RAISING STANDARDS Assessment and Recommendations. OECD, 2014 (https://read.oecd.org/10.1787/97892642258 17-en?format=pdf)

16 Niimura J, Nakanishi M, Yamasaki S, Nishida A. Regional supply of outreach service and length of stay in psychiatric hospital among patients with schizophrenia: national case mix data analysis in Japan. Psychiatry Res 2017; 258: 295-8.

17 Noda $T$, Sugiyama $N$, Sato $M$, Ito $H$, Sailas E, Putkonen $H$, et al. Influence of patient characteristics on duration of seclusion/restrain in acute psychiatric settings in Japan. Psychiatry Clin Neurosci 2013; 67: 405-11.

18 Tachimori H, Takeshima T, Kono T, Akazawa M, Zhao X. Statistical aspects of psychiatric inpatient care in Japan: based on a comprehensive nationwide survey of psychiatric hospitals conducted from 1996 to 2012. Psychiatry Clin Neurosci 2015; 69: 512-22.

19 McGrnahan R, Rennick-Egglestone S, Ramsay A, Llewellyn-Beardsley J, Bradstreet S, Callard F, et al. Curation of mental health recovery narrative collections: systematic review and qualitative synthesis. JMIR Ment Health 2019; 6: e14233.

20 Ellison ML, Belanger LK, Niles BL, Evans LC, Bauer MS. Explication and definition of mental health recovery: a systematic review. Adm Policy Ment Health 2018; 45: 91-102.

21 GBD 2017 Disease and Injury Incidence and Prevalence Collaborator. Global, regional, and national incidence, prevalence, and years lived with disability for 354 diseases and injuries for 195 countries and territories, 1990-2017: a systematic analysis for the Global Burden of Disease Study 2017. Lancet 2018; 392: 1789-858.

22 National Collaborating Centre for Mental Health. Working Well Together: Evidence and Tools to Enable Co-Production in Mental Health Commissioning. National Collaborating Centre for Mental Health, 2019 (http://rcpsych.ac.uk/ docs/default-source/improving-care/nccmh/working-well-together/workingwell-together-evidence-and-toolsto-enable-coproduction-in-mental-healthcommissioning.pdf).

23 Biernacki P, Waldorf D. Snowball sampling: problems and techniques of chain referral sampling. Sociol Methods Res 1981; 10: 141-63.

24 Nakanishi M, Tanaka S, Kurokawa G, Ando S, Yamasaki S, Fukuda M, et al. Inhibited autonomy for promoting physical health: qualitative analysis of narratives from persons living with severe mental illness. BJPsych Open 2019; 5: e10. 
25 Niimura J, Tanoue M, Nakanishi M. Challenges following discharge from acute psychiatric inpatient care in Japan: patients' perspectives. J Psychiatr Ment Health Nurs 2016; 23: 576-84.

26 Braun V, Clarke V. Using thematic analysis in psychology. Qual Res Psychol 2006; 3: 77-101.

27 Link BG, Struening EL, Neese-Todd S, Asmussen S, Phelan JC. Stigma as a barrier to recovery: the consequences of stigma for the self-esteem of people with mental illnesses. Psychiatr Ser 2001; 52: 1621-6.

28 Jas E, Wieling M. Providers' competencies positively affect personal recovery of involuntarily admitted patients with severe mental illness: a prospective observational study. Int J Soc Psychiatry 2018; 64: 145-55.

29 Kowalski MA. Mental health recovery: the effectiveness of peer servicers in the community. Community Ment Health J 2019; 56: 568-80.

30 Davidson L, Bellamy C, Guy K, Miller R. Peer support among persons with severe mental illnesses: a review of evidence and experience. World Psychiatry 2012; 11: 123-8.
31 Keet R, de Vetten-Mc Mahon M, Shields-Zeeman L, Ruud T, van Weeghel J, Bahler M, et al. Recovery for all in the community; position paper on principles and key elements of community-based mental health care. BMC Psychiatry 2019; 19: 174.

32 Bryson SA, Gauvin E, Jamieson A, Rathgeber M, Faulkner-Gibson L, Bell S, et al. What are effective strategies for implementing trauma-informed care in youth inpatient psychiatric and residential treatment settings? A realist systematic review. Int J Ment Health Syst 2017; 11: 36.

33 Johnson $\mathrm{T}$, Kulesa $\mathrm{P}, \mathrm{Cho} \mathrm{YI}$, Shavitt $\mathrm{S}$. The relation between culture and response styles: evidence from 19 countries. J Cross Cult Psychol 2005; 36: 264-77. 